\title{
Novel method of detecting H1N1 using microwaves
}

\author{
Anil Lonappan \\ Department of Electrical Engineering, Mangosuthu University of Technology, Durban, South Africa \\ Email: lonappan@mut.ac.za
}

Received 31 May 2012; revised 28 June 2012; accepted 13 July 2012

\begin{abstract}
H1N1 virus is a subtype of influenza A virus and was the most common cause of human influenza flu in 2009. This paper presents a new method of detecting H1N1. The dielectric properties respiratory mucus is studied at microwave frequencies using rectangular cavity perturbation technique at the S-band with the different samples of respiratory mucus obtained from healthy donors as well as from patients suffering from H1N1. It is observed that an appreciably variation is found in the dielectric properties of patient samples as that of normal healthy samples and this measurements were in good agreement with the clinical analysis. This measurement technique is quick, simple and suggests a new novel method of diagnosing H1N1 using microwaves.
\end{abstract}

Keywords: Mucus; H1N1; Cavity Perturbation; Clinical Analysis

\section{INTRODUCTION}

A novel strain of influenza A (H1N1) spread rapidly through Mexico in April 2009 and now spans the globe. By the time the World Health Organization (WHO) was notified and had responded, geographical containment was not feasible, leading the agency to call for mitigation. Early indications are that the first wave may not be as widespread or pathogenic as originally feared, but this influenza strain could evolve to become more dangerous in subsequent waves [1]. In June 2009, the World Health Organization declared the new strain of swine-origin H1N1 as a pandemic. Influenza pandemics occur when human populations are infected by a variant virus to which a population has no prior immunity. The influenza A viruses that infect mammals like us replicate principally in the epithelial cells of the airways [2]. Complications of influenza are well-documented, including abdominal pain, nausea, vomiting, diarrhea, bronchiolitis, pneumonia, as well as neurological manifestations such as acute encephalopathy and encephalitis [3]. The crucial genes are those encoding the two viral surface proteins hemagglutinin (H or HA) and neuraminidase (N or NA). The HA facilitates viral entry by binding to sialic acid residues on the epithelial cell surface, while the NA functions to cleave such attachments, and so release new virus particles, or virions, both from the cell and from the slimy mucous that protects the lung and trachea. The new virions are then free to spread the infection, both from cell to cell and to other susceptible individuals. Antibodies that bind to either the HA or the NA and block their function effectively prevent (or terminate) the infectious process and thus provide protective immunity. A number of different laboratory diagnostic tests can be used for detecting the presence of influenza viruses in respiratory specimens, including direct antigen detection tests, virus isolation in cell culture, or detection of influenza-specific RNA by real-time reverse transcriptasepolymerase chain reaction (RT-PCR). These tests differ in their sensitivity and specificity in detecting influenza viruses as well as in their commercial availability, the amount of time needed from specimen collection until results are available. This communication presents a new method of detecting H1N1 by measuring dielectric properties of respiratory mucus collected from healthy donors as well as from patients suffering from H1N1.

Medical science has advanced greatly in the last century, there is still much that is not understood about the way the human body interacts with many things, including microwaves. The potential for medical uses of microwaves lies in two areas therapeutic and diagnostic field and many new medical microwave devices are also developed [4,5]. All the known therapeutic uses of microwaves involve the heating of tissue. Carefully controlled, microwave-generated heat can have a therapeutic effect on a number of ailments. Microwave medical devices ability depends upon how far microwaves can deeply penetrate into living tissues. The depth to which microwaves can penetrate tissues is primarily a function of the dielectric properties of tissues and of the frequency of the microwaves. Lower the water content of the tissue the deeper a wave at a given frequency and lower the frequency the deeper is the depth of penetration into tissues with given water content. Thus, there is a need to 
study the interaction of microwave with tissues especially its effect on biological materials. The key element in the microwave study is the determination of the absorbed energy. The amount of energy absorbed is a function of the complex permittivity of a material [6]. Hence, it is crucial to know the dielectric properties of biological materials and the various constituents thereof. Exhaustive studies of dielectric parameters of various human tissues and body fluids at different RF frequencies have been reported [7-9]. Different measurement techniques are used to measure the complex permittivity of a material and the chosen technique depends on various factors such as the nature of the sample and the frequency range used [10-13]. When only very small volumes of the sample are available, the cavity perturbation technique is an attractive option as it requires only minute volumes for the measurement [14]. This makes it suitable for the dielectric study of respiratory mucus because very small volume need only be extracted by this procedure. The rectangular cavity perturbation technique has been employed for the measurement of the dielectric parameters of respiratory mucus samples obtained from healthy persons as well as from patients with H1N1 in this work, in the frequency range 2 to $3 \mathrm{GHz}$. It is noticed that a remarkable change in the dielectric properties of $\mathrm{H} 1 \mathrm{~N} 1$ samples with the normal healthy samples and these measurements were in good agreement with clinical analysis. This microwave measurement procedure is simple and extraction of mucus from persons is least painful and nonsurgical in nature. These results prove a new novel method of diagnosing H1N1 using microwaves.

\section{SAMPLE PREPARATION}

The respiratory mucus samples were collected by using aspirator from donors as well as from patients. Then it is sealed in sample holders at $5^{\circ} \mathrm{C}$ to maintain their viability. Measurements were carried out on samples which were less than one day old.

\section{EXPERIMENTAL SET UP}

The experimental set-up consists of a transmission type S-band rectangular cavity resonator, HP 8714 ET network analyser as in Figures 1 and 2. The cavity resonator is a transmission line with one or both ends closed. The numbers of resonant frequencies are determined by the length of the resonator. The resonator in this set-up is excited in the $\mathrm{TE}_{10 \rho}$ mode. The sample holder which is made of glass in the form of a capillary tube flared to a disk shaped bulb at the bottom is placed into the cavity through the non-radiating cavity slot, at broader side of the cavity which can facilitate the easy movement of the holder. The resonant frequency $f_{o}$ and the corresponding quality factor $Q_{o}$ of the cavity at each resonant peak with the empty sample holder placed at the maximum electric field are noted. The same holder filled with known amount of sample under study is again introduced into the cavity resonator through the non-radiating slot. The resonant frequency of the sample loaded cavity is selected and the position of the sample is adjusted for maximum perturbation (i.e. maximum shift of resonant frequency with minimum amplitude for the peak). The new resonant frequency $f_{s}$ and the quality factor $Q_{s}$ are noted. The same procedure is repeated for other resonant frequencies.

\section{THEORY}

When a material is introduced into a resonant cavity, the cavity field distribution and resonant frequency are changed which depend on shape, electromagnetic properties and its position in the fields of the cavity. Dielectric material interacts only with electric field in the cavity.

According to the theory of cavity perturbation, the complex frequency shift is related as [14]

$$
\begin{gathered}
-\frac{\mathrm{d} \Omega}{\Omega} \approx \frac{\left(\bar{\varepsilon}_{r}-1\right) \int_{V_{s}} E \cdot E_{0}^{*} \mathrm{~d} V}{2 \int_{V_{c}}\left|E_{0}\right|^{2} \mathrm{~d} V} \\
\frac{\mathrm{d} \Omega}{\Omega} \approx \frac{\mathrm{d} \omega}{\omega}+\frac{j}{2}\left[\frac{1}{Q_{s}}-\frac{1}{Q_{0}}\right]
\end{gathered}
$$

Equating (1) and (2) and separating real and imaginary parts results

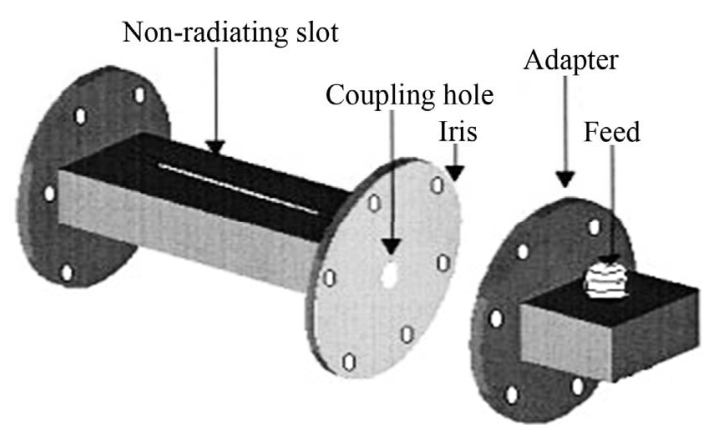

Figure 1. Schematic diagram of the rectangular cavity.

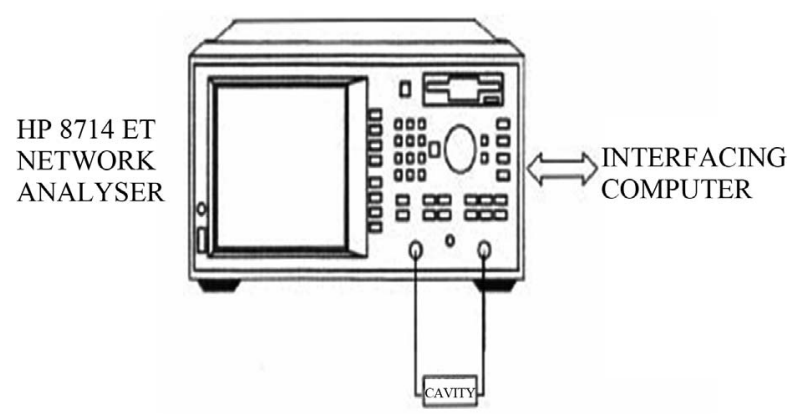

Figure 2. Schematic diagram of the measurement setup. 


$$
\begin{gathered}
\varepsilon_{r}^{\prime}-1=\frac{f_{o}-f_{s}}{2 f_{s}}\left(\frac{V_{c}}{V_{s}}\right) \\
\varepsilon_{r}^{\prime \prime}=\frac{V_{c}}{4 V_{s}}\left(\frac{Q_{o}-Q_{s}}{Q_{o} Q_{s}}\right)
\end{gathered}
$$

Here, $\bar{\varepsilon}_{r}=\varepsilon_{r}^{\prime}-j \varepsilon_{r}^{\prime \prime}, \varepsilon_{r}$ is the relative complex permittivity of the sample, $\varepsilon_{r}^{\prime}$ is the real part of the relative complex permittivity, which is known as dielectric constant. $\varepsilon_{r}^{\prime \prime}$ is the imaginary part of the relative complex permittivity associated with the dielectric loss of the material. $V_{s}$ and $V_{c}$ are corresponding volumes of the sample and the cavity resonator. The conductivity can be related to the imaginary part of the complex dielectric constant as

$$
\sigma_{e}=\omega \varepsilon^{\prime \prime}=2 \pi f \varepsilon_{0} \varepsilon_{r}^{\prime \prime}
$$

\section{RESULTS AND DISCUSSION}

The microwave studies of the respiratory mucus obtained from healthy donors as well as from patients suffering from $\mathrm{H} 1 \mathrm{~N} 1$ were done using cavity perturbation technique and the results are shown in Figures $\mathbf{1}$ and 2. Clinical evaluation of these samples was also done simultaneously and the results are tabulated in Tables $\mathbf{1}$ and 2. The Figure 3 shows the variation of the dielectric constants in normal respiratory mucus and H1N1 samples. It is observed that the H1N1 samples exhibit a higher dielectric constant than that of the normal respiratory mucus samples. Figure 4 shows the variation of the conductivity in normal respiratory mucus and H1N1

Table 1. Variation glucose level in normal respiratory mucus samples and H1N1 samples.

\begin{tabular}{cl}
\hline $\mathrm{mN}-1$ & $11-15 \mathrm{mg} / \mathrm{dL}$ (Normal sample) \\
$\mathrm{mN}-2$ & $13-19 \mathrm{mg} / \mathrm{dL}$ \\
$\mathrm{mN}-3$ & $12-18 \mathrm{mg} / \mathrm{dL}$ \\
$\mathrm{mN}-4$ & $12-17 \mathrm{mg} / \mathrm{dL}$ \\
$\mathrm{mH}-1$ & $08-10 \mathrm{mg} / \mathrm{dL}(\mathrm{H} 1 \mathrm{~N} 1 \mathrm{sample})$ \\
$\mathrm{mH}-2$ & $03-08 \mathrm{mg} / \mathrm{dL}$ \\
$\mathrm{mH}-3$ & $05-09 \mathrm{mg} / \mathrm{dL}$ \\
$\mathrm{mH}-4$ & $02-07 \mathrm{mg} / \mathrm{dL}$
\end{tabular}

Table 2. Variation of constituents in normal respiratory mucus samples and H1N1 samples.

\begin{tabular}{lcc}
\hline & $\begin{array}{c}\text { Normal Range } \\
\text { (mEq/liter) }\end{array}$ & H1N1 Range (mEq/liter) \\
\hline Hemagglutinin & 0.01 to 0.03 & 0.001 to 0.002 \\
Neuraminidase & 0.01 to 0.02 & 0.001 to 0.002 \\
$\quad$ Mucin & 0.002 to 0.011 & 0.001 to 0.013 \\
Inorganic salts & 0.4 to 0.72 & 0.22 to 0.34 \\
\hline
\end{tabular}

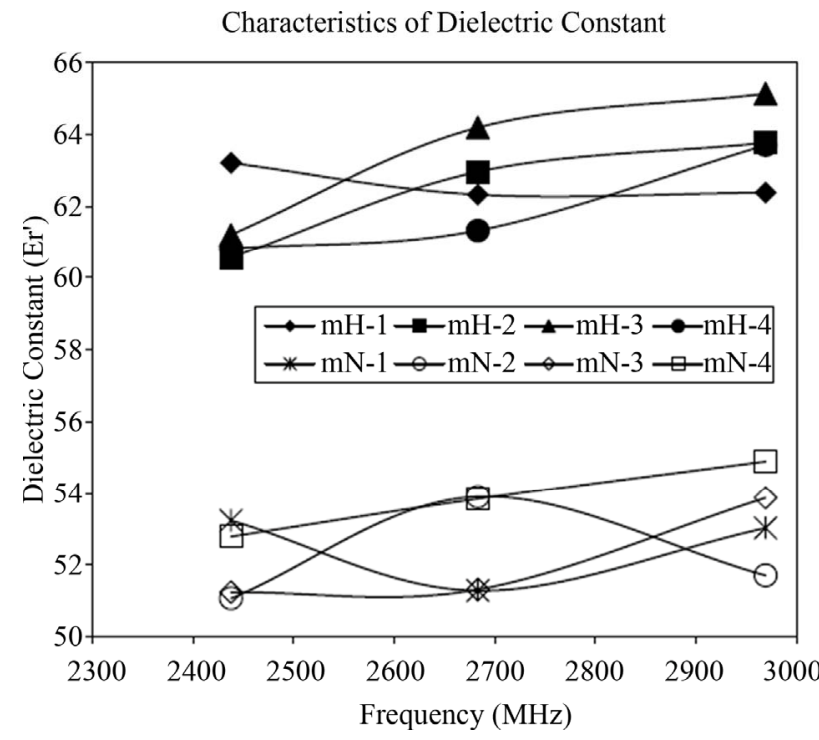

Figure 3. Variation of dielectric constant in normal respiratory mucus samples and H1N1 samples.

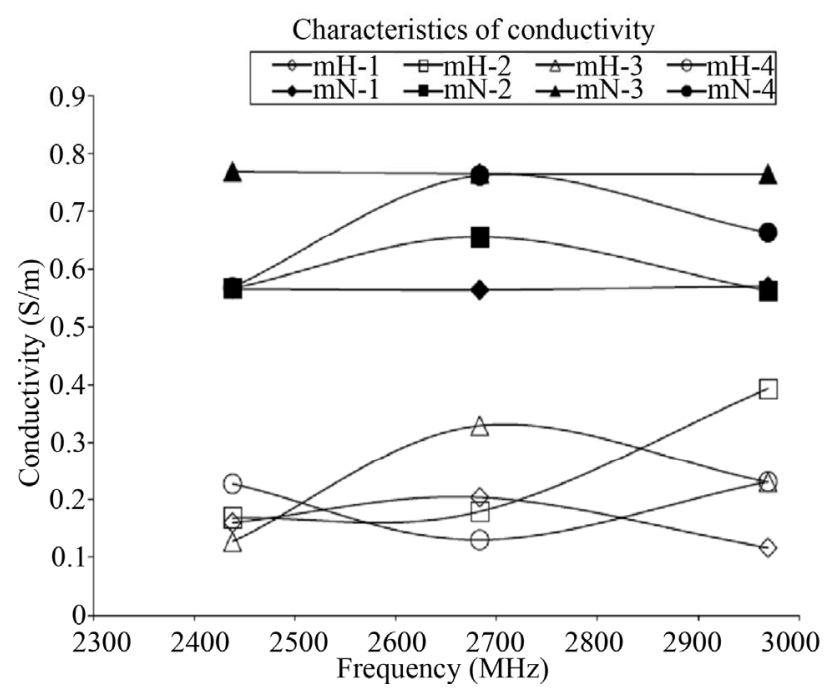

Figure 4. Variation of conductivity in normal respiratory mucus samples and H1N1 samples.

mucus samples. It can be found that distinct variation in the conductivities between H1N1 mucus samples and normal samples. This increased conductivity is by the presence of higher level of glucose as well as inorganic salts. Thus in the specified band of frequencies, in normal respiratory mucus samples and $\mathrm{H} 1 \mathrm{~N} 1$ samples exhibit distinct variation of dielectric constant and conductivity with frequency and is in good agreement with clinical analysis.

\section{CONCLUSION}

The microwave characterization of the in normal mucus samples and H1N1 samples is done using cavity perturbation technique. This method is adopted as it requires 
very low volume of sample and hence applicable to samples like mucus. It is observed that an appreciably change in the dielectric properties of patient samples with the normal healthy samples and this measurements were in good agreement with clinical analysis. This measurement technique is quick, simple and suggests a new novel method of diagnosing H1N1 using microwaves.

\section{REFERENCES}

[1] Chan, M. (2009) Influenza A(H1N1). http://www.who.int/mediacentre/news/statements/2009/h 1n1_20090429/en/index.html

[2] Salomon, R. and Webster, R.G. (2009) The influenza virus enigma. Cell, 136, 402-410. doi:10.1016/j.cell.2009.01.029

[3] Morishima, T., Togashi, T., Yokota, S., Okuno, Y., Miyazaki, C., Tashiro, M. and Okabe, N. (2002) Encephalitis and encephalopathy associated with an influenza epidemic in Japan. Clinical Infectious Diseases, 35, 512517. doi:10.1086/341407

[4] Rosen, A. and Rosen, H.D., (1995) New frontiers in medical device technology. Wiley, New York.

[5] Rosen, A., Vorst, A. and Kotsuka, Y. (2000) Mini-special issue on $\mathrm{RF} /$ microwave applications in medicine. IEEE Transactions on Microwave Theory Technique, 48.

[6] Von Hippel, A. (1995) Dielectric and waves. Artech House, London.

[7] Gabriel, S., Lau, R.W. and Gabriel, C. (1996) The dielectric properties of biological tissues: II. Measurements on the frequency range $10 \mathrm{~Hz}$ to $20 \mathrm{GHz}$, literature survey. Physics Medicine Biology, 41, 2251-2269.

\section{doi:10.1088/0031-9155/41/11/002}

[8] Cook, H.F. (1951) Dielectric behavior of human blood at microwave frequencies. Nature, 168, 247-248. doi: $10.1038 / 168247 \mathrm{a} 0$

[9] Cook, H.F. (1951) The dielectric behavior of some types of human tissues at microwave frequencies. British Journal of Applied Physics, 2, 295-300. doi:10.1088/0508-3443/2/10/304

[10] Ghodgaonkar, D.K., Varadan, V.V. and Varadan, V.K. (1990) Free space measurement of complex permittivity and complex permeability of magnetic materials at microwave frequencies. IEEE Transactions on Instrumentation and Measurement, 19, 387-394. doi: $10.1109 / 19.52520$

[11] Ghodgaonkar, D.K., Varadan, V.V. and Varadan, V.K. (1989) A free space method for measurement of dielectric constant and loss tangents at microwave frequencies. IEEE Transaction on Instrumentation and Measurement, 38, 789-793. doi:10.1109/19.32194

[12] Barry, W. (1986) A broadband, automated, stripline technique for the simultaneous measurement of complex permittivity and complex permeability. IEEE Transaction on Microwave Theory and Techniques, 34, 80-84. doi:10.1109/TMTT.1986.1133283

[13] Abbas, Z., Pollard, R.D. and Kelsall, R.W. (1998) A rectangular dielectric waveguide technique for determination of permittivity of materials at w-band. IEEE Transactions on Microwave Theory and Techniques, 46, 2011-2015. doi:10.1109/22.739275

[14] Mathew, K.T. (2005) Perturbation theory. Encyclopedia of RF and Microwave Engineering, 4, 3725-3735. 